\title{
Enhanced magnetic field sensitivity of spin-dependent transport in cluster-assembled metallic nanostructures
}

\author{
SANTIAGO SERRANO-GUISAN ${ }^{1}$, GIULIA DI DOMENICANTONIO², MOHAMED ABID ${ }^{1}$, JEAN-PIERRE ABID³, \\ MATTHIAS HILLENKAMP2 ${ }^{2}$, LAURENT GRAVIER ${ }^{1}$, JEAN-PHILIPPE ANSERMET ${ }^{1 *}$ AND CHRISTIAN FÉLIX2* \\ ${ }^{1}$ Laboratoire de Physique des Matériaux Nanostructurés, Ecole Polytechnique Fédérale de Lausanne, CH-1015 Lausanne-EPFL, Switzerland \\ ${ }^{2}$ Laboratoire des Nanostructures en Matrices, Ecole Polytechnique Fédérale de Lausanne, CH-1015 Lausanne-EPFL, Switzerland \\ ${ }^{3}$ Laboratoire d'Electrochimie Physique et Analytique, Ecole Polytechnique Fédérale de Lausanne, CH-1015 Lausanne-EPFL, Switzerland \\ *e-mail: jean-philippe.ansermet@epfl.ch; christian.felix@epfl.ch
}

Published online: 13 August 2006; doi:10.1038/nmat1713

The emerging field of spintronics explores the many possibilities offered by the prospect of using the spin of the electrons for fast, nanosized electronic devices. The effect of magnetization acting on a current is the essence of giant or tunnel magnetoresistance. Although such spintronics effects already find technological applications, much of the underlying physics remains to be explored. The aim of this article is to demonstrate the importance of spin mixing in metallic nanostructures. Here we show that magnetic clusters embedded in a metallic matrix exhibit a giant magnetic response of more than $500 \%$ at low temperature, using a recently developed thermoelectric measurement. This method eliminates the dominating resistivity component of the magnetic response and thus reveals an intrinsic spin-dependent process: the conduction-electron spin precession about the exchange field as the electron crosses the clusters, giving rise to a spin-mixing mechanism with strong field dependence. This effect appears sensibly only in the smallest clusters, that is, at the level of less than 100 atoms per cluster. pintronics seeks to exploit the interplay of spin-polarized conduction electrons and magnetization in nanostructures. Spin-dependent scattering leads to giant magnetoresistance ${ }^{1-5}$ (GMR) and tunnelling magnetoresistance $e^{6-8}$, whereas the converse effect of a spin-polarized current on the magnetization ${ }^{9-11}$ can be taken advantage of in magnetoresistive memory bits ${ }^{12}$ and gigahertz oscillators $^{13}$. GMR as a field sensing measurement of a resistivity ratio $\Delta R / R$ is dominated by non-magnetic and spin-independent scattering processes determining $R$. Instead, the thermoelectric measurement protocol developed in our laboratory ${ }^{14}$ depends on the first derivative of $R$ with respect to the temperature and thus suppresses this resistive contribution. This allows us to fully reveal the otherwise negligible spin-mixing processes. In multilayers this mechanism is essentially a spin-dependent Peltier effect that roughly doubles the field sensitivity compared with $\mathrm{GMR}^{14}$. Here we have applied this measurement protocol to granular clusterassembled materials ${ }^{15}$, the geometry of which is not appropriate for a Peltier effect. Hence a clearly different microscopic mechanism takes a predominant role here. We invoke the predominance of spin mixing caused by a spin-precession effect ${ }^{16}$ that is completely different in nature. Spin mixing was predicted to decrease GMR responses, as it scrambles the two spin channels of conduction. In our measurement scheme, on the contrary, it results in a 100 -fold increase of the field response compared with GMR. The combined use of cluster-assembled materials and a novel measurement method thus reveal a different spin transport effect and may open a new route towards possible applications.

Samples were prepared (see the 'Methods' section) according to the strategy of 'cluster-assembled materials' (Fig. 1). Briefly, the samples consist of thin films of copper in which well-defined cobalt clusters are dispersed. Narrow distributions of metal cluster ions are prepared and analysed in the gas phase and are co-deposited with the copper matrix onto a substrate of polyimide. This method allows for the simultaneous control of the cluster size and their concentration. Sample thickness and Co loading were kept constant 

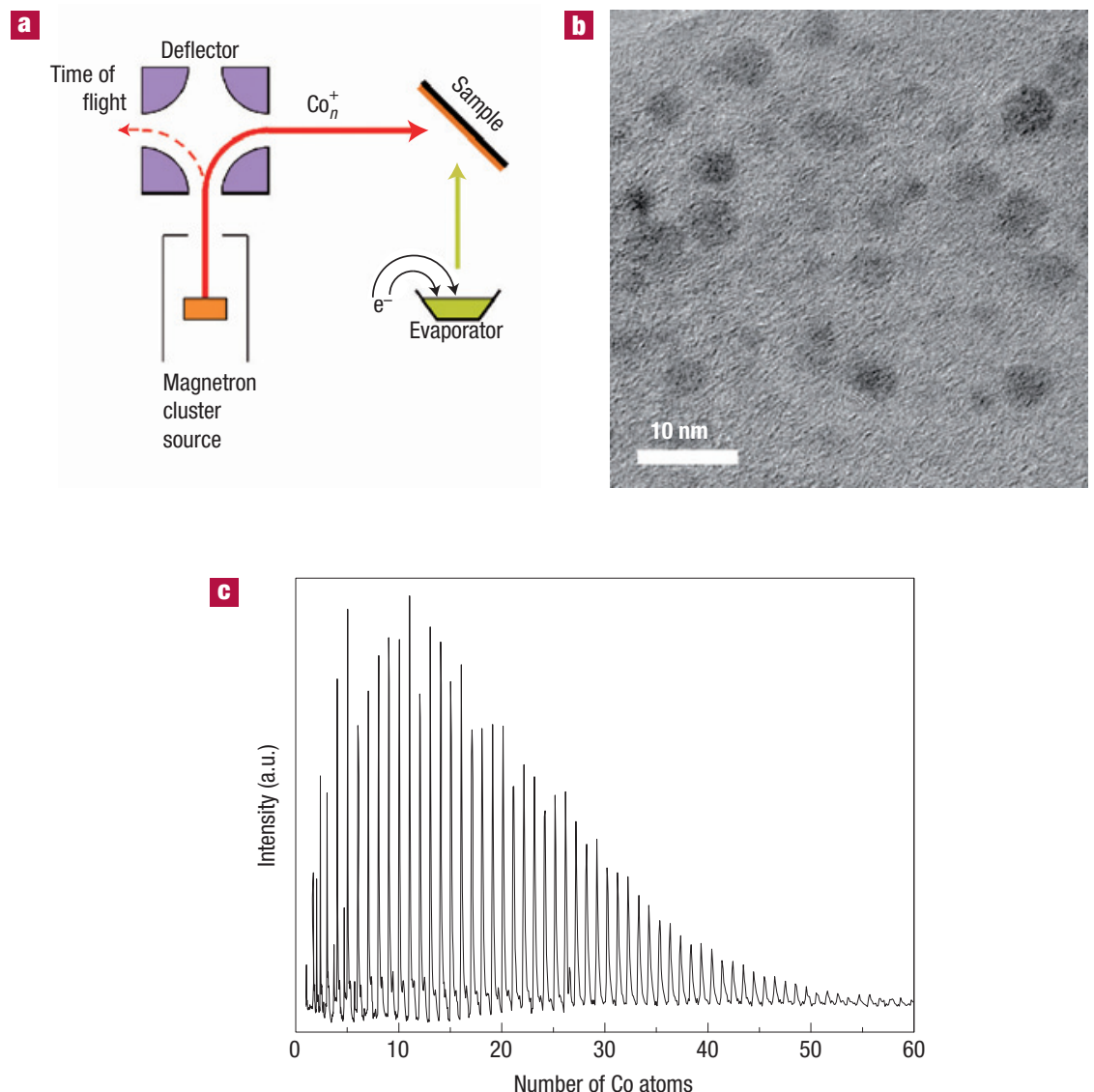

Figure 1 Cluster-assembled material. a, Co cluster ions produced with a magnetron cluster source are directed towards a sample holder. By changing the source parameters, the cluster size can be adjusted over a wide range. Simultaneously Cu is evaporated from a crucible and deposited on the same sample holder. $\mathbf{b}$, Transmission electron micrograph of the sample with $\langle n\rangle=6,500$. c, The cluster ion size distribution can be monitored with a time-of-flight mass spectrometer; a typical spectrum is given for $\mathrm{Co}_{n}^{+}$with $\langle n\rangle=15$. The small satellite peaks are $\mathrm{Co}_{n} \mathrm{Ar}^{+}$complexes.

at $50 \mathrm{~nm}$ and $8 \%$, respectively. The mean sizes of the embedded clusters used in this study were $\langle n\rangle=15 ; 600 ; 2,300$ and 6,500 atoms per cluster.

Besides conventional resistance measurements, we carried out a thermoelectric experiment that measures the thermogalvanic voltage (TGV) and that was initially developed for multilayer systems $s^{14,17}$. The principle of this experiment is shown in Fig. 2a, a chopped laser diode shines on the film and induces a temperature oscillation of about $1 \mathrm{~K}$. The laser irradiates a large portion of the film and does not induce a net temperature gradient. The corresponding voltage across the whole film is measured by lock-in detection under a constant charge current $I_{\text {d.c. }}$. Although there could also be a contribution from the derivative of the resistance with respect to temperature, this effect, however, is negligible in metals below $20 \mathrm{~K}$, where the resistance is temperature independent. Consequently, TGV measurements carried out at $14 \mathrm{~K}$ (Fig. 2b) are independent of the temperature dependence of the resistance.

Magnetoresistance and magnetothermogalvanic voltage (MTGV) measurements were carried out on samples of four different cluster sizes at $14 \mathrm{~K}$ under an externally applied magnetic field of up to $0.8 \mathrm{~T}$. The magnetic response of the resistance is in the range of $1-6 \%$ (Figs 3b, 4a), which is coherent with GMR processes in such granular nanostructures ${ }^{4}$. In contrast, the MTGV response of the sample of 15 atoms per cluster shows an extraordinary large magnetic response of $500 \%$ at $0.8 \mathrm{~T}$ (Fig. 3a). A low-temperature response of this magnitude, about 120 times the GMR ratio, is observed for different samples and all field orientations. Its amplitude at larger fields and lower temperatures is expected to be even higher as saturation is not reached at $0.8 \mathrm{~T}$.

We infer from several observations that MTGV measures a spindependent transport mechanism different from GMR. The MTGV amplitude drops sharply with increasing grain size (Fig. 4a), and quasi-vanishes for the sample of 6,500 atoms per cluster. This differs from the moderate size dependence of GMR (Fig. 4b). Moreover, the MTGV response exhibits saturation at $\sim 0.2 \mathrm{~T}$ for 600 atoms per cluster, whereas none of the GMR curves show saturation at $0.8 \mathrm{~T}$. Finally, we observe that the MTGV vanishes with increasing temperature, whereas some residual GMR is retained at room temperature (see Supplementary Information, Fig. S1).

A key parameter for the analysis of the MTGV is the intergranular distance. Figure $4 \mathrm{c}$ shows the microscopic distribution of Co clusters in a copper matrix for the different cluster sizes when the atomic concentration is fixed at $8 \%$. A simple estimate yields a mean intercluster distance of about three cluster diameters (centre to centre). As a consequence, large copper paths open up in the case of larger clusters. This is of limited importance for GMR, because the mean distance between two cobalt clusters is always below the spin diffusion length of copper $\left(l_{\mathrm{sf}}^{\mathrm{Cu}} \sim 40-60 \mathrm{~nm}\right)^{18,19}$. 

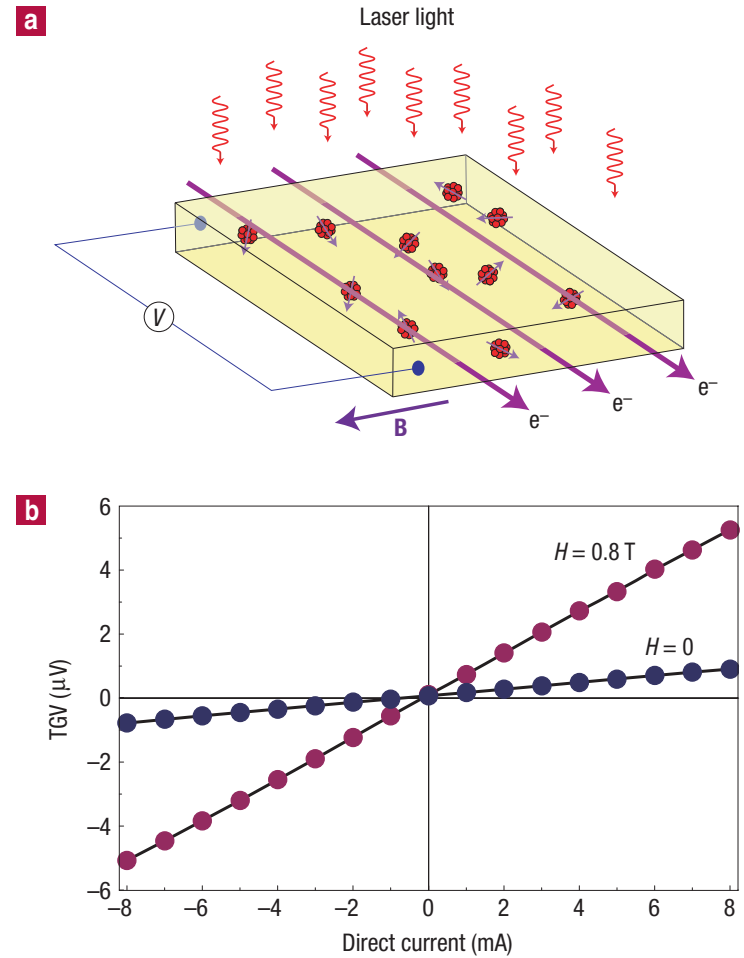

Figure 2 TGV experiment. a, The voltage drop $V$ at the ends of a strip of film in which a constant current $I_{\text {d.c. }}$ flows is detected in phase with the temperature oscillation caused by a chopped laser light. $\mathbf{b}$, TGV signal of the sample with $\langle n\rangle=15$ atoms per cluster, measured at $14 \mathrm{~K}$ and plotted as a function of $I_{\text {d.c. }}$. A linear dependence is observed for both zero and non-zero external magnetic fields.

To focus the attention on identifying the underlying spindependent mechanism, we treat the sample as a homogeneous effective medium. We use a simple thermodynamic argument to show that spin mixing is at the origin of the huge field dependence of the TGV. We analyse the spin-dependent transport in terms of the two-current model ${ }^{20}$. We state that the currents $j_{\uparrow, \downarrow}$ are linearly related to their associated generalized forces $\nabla \bar{\mu}_{\downarrow, \uparrow}$, which are the gradients of their electrochemical potentials. With the usual parameter $\beta$ to express the spin asymmetry of the conductivities $\sigma_{\uparrow(\downarrow)}=(1 / 2) \sigma(1 \pm \beta)$, the transport matrix is:

$$
\left(\begin{array}{l}
j_{\uparrow} \\
j_{\downarrow}
\end{array}\right)=-\frac{1}{q}\left(\begin{array}{cc}
(\sigma / 2)(1+\beta) & L_{\uparrow \downarrow} \\
L_{\downarrow \uparrow} & (\sigma / 2)(1-\beta)
\end{array}\right)\left(\begin{array}{l}
\nabla \bar{\mu}_{\uparrow} \\
\nabla \bar{\mu}_{\downarrow}
\end{array}\right),
$$

where $q$ is the charge of an electron and $\sigma$ is the isothermal electric conductivity. We introduce the non-diagonal transport parameters $L_{\uparrow \downarrow}$ and $L_{\downarrow \uparrow}$ that express the mixing of the spin currents. By setting as a boundary condition that the polarization current $j_{\uparrow}-j_{\downarrow}$ vanishes in the gold leads, we establish an effective conductivity:

$$
\sigma_{\text {eff }}=\sigma\left(1-\beta^{2}\right)\left(1+\frac{\Delta L^{2}}{\sigma^{2}}\right) .
$$

The first two terms correspond to the usual GMR effect. In this effective medium approximation, the value of $\beta$ must be considered as dependent on the degree of orientation or misalignment of the magnetization of successive grains. The last term contains $\Delta L=L_{\uparrow \downarrow}-L_{\downarrow \uparrow}$, that is, the difference in spin-mixing rates

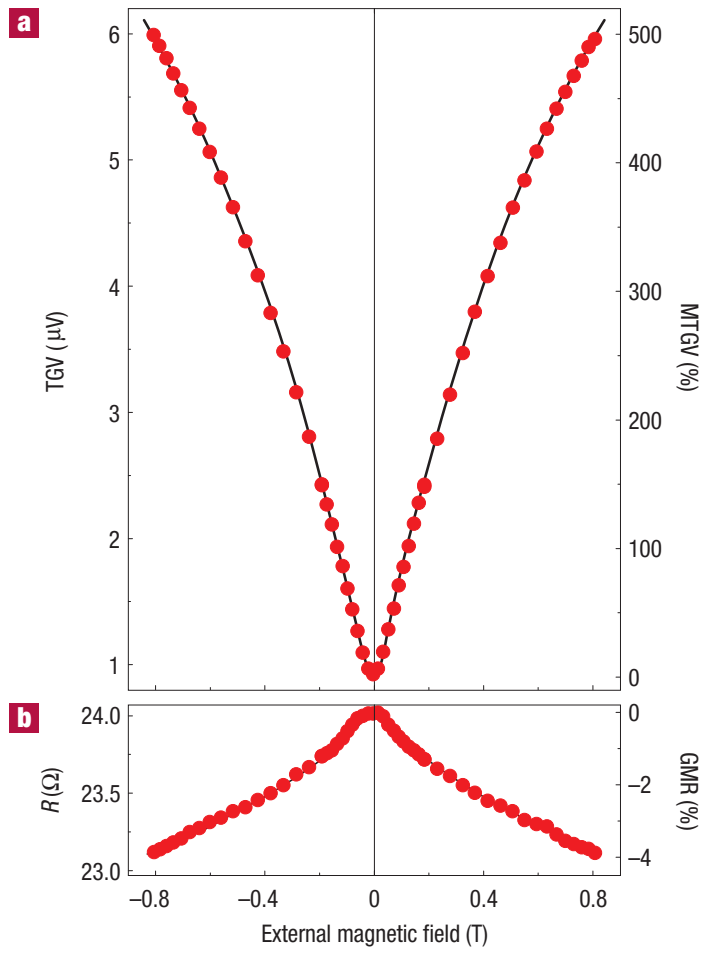

Figure 3 MTGV data. a, TGV measurements carried out at $14 \mathrm{~K}$ with $I_{\text {d.c. }}=8 \mathrm{~mA}$ on the sample of $\langle n\rangle=15$ atoms per cluster, and plotted as a function of the external applied magnetic field. The magnetic response reaches $500 \%$ at $0.8 \mathrm{~T}$.

b, Magnetoresistive measurements carried out under the same conditions.

going from majority to minority spins and vice versa. The TGV experiment measures the first derivative of $\sigma_{\text {eff }}$ with respect to the temperature. At $14 \mathrm{~K}$, the magnetic response of $\sigma\left(1-\beta^{2}\right)$ is only a few per cent. Therefore, the amplitude of the MTGV must arise quasi-exclusively from the spin-mixing term in equation (1).

The main spin-mixing mechanism is usually assumed to be electron-magnon collisions ${ }^{21,22}$. However, given the size of the Co clusters considered in this study, scattering of conduction electrons by low- $q$ magnons seems unlikely $y^{23}$ and another spin-mixing mechanism must be considered. Following earlier work $^{16}$, we propose that in granular materials, for which the grain size is much smaller than the spin diffusion length, the spins of the conduction electrons precess about the exchange field as they pass through the magnetic grains. This mechanism was named the 'jitterbug spin channel mixing ${ }^{24}$. Indeed the precession about the exchange field is equivalent to transitions between the two spin channels (for example, see ref. 25), that is, to a spin-mixing effect (Fig. 5a). At zero field, the random orientation of magnetic moments in each cluster yields a 'jitterbug' spin mixing that is completely symmetric $(\Delta L=0)$, corresponding to a minimum TGV signal. As the magnetic field increases, the ordering of the magnetic moments induces a splitting of the spin-up and spin-down levels quantized in the direction of the magnetic field. According to the basic principles of magnetic resonance, the fluctuating magnetic field as perceived in the reference frame of a moving electron can produce a relaxation of its spin. The transition rates of the up- to the downstate $\left(L_{\uparrow \downarrow}\right)$ and that of the converse process are not equal (Fig. $5 \mathrm{~b}$ ). Their ratio is given by the ratio of populations of the down- and up-states ${ }^{26}$. In other words, to the extent that we expect a spin 

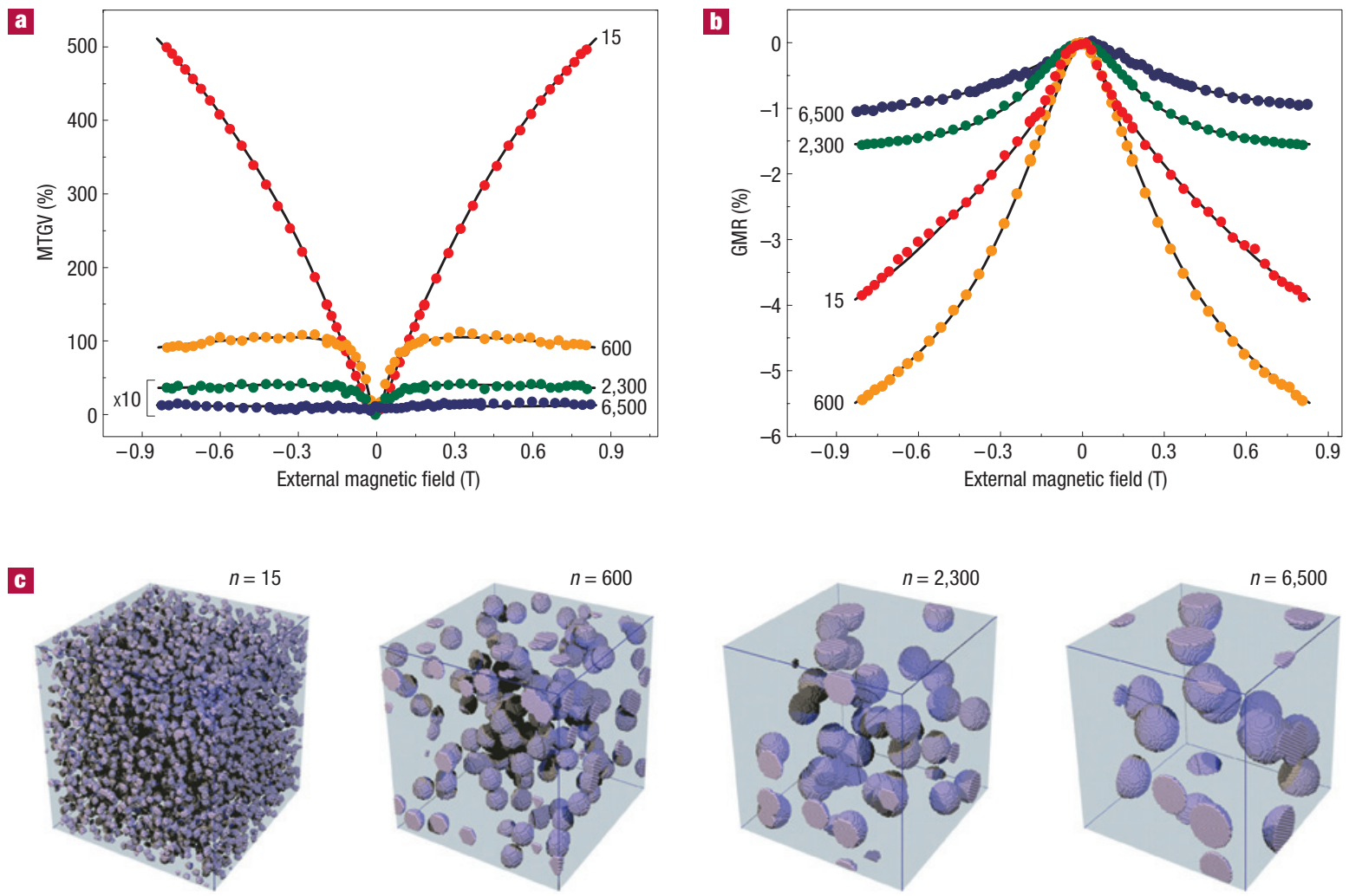

Figure 4 Cluster size dependence. a,b, MTGV (a) and magnetoresistive (b) measurements carried out on samples of different cluster size under the same conditions as in Fig. 3. c, Visualization in a cube of $12.6 \mathrm{~nm}$ per side of Co clusters of size $n$ randomly distributed in a face-centred-cubic lattice. All remaining lattice sites are occupied by $\mathrm{Cu}$ atoms (not shown). The relative number of $\mathrm{Co}$ atoms is set to $8 \%$ in all images.

polarization, we must also expect $\Delta L \neq 0$. This mechanism is very sensitive to intergranular distances and to the relative orientations of magnetic moments in neighbouring clusters.

Thus, our effective medium model offers a qualitative explanation to describe the data. The spin-mixing mechanism suggested here is effective only for small cluster systems, for which the current flows through the magnetic cobalt grains; for larger clusters, the current flow in the $\mathrm{Cu}$ matrix dominates and the MTGV vanishes. The prospects for room-temperature application are favourable, MTGV at room temperature has already been observed in nanowires ${ }^{17}$. A detailed analysis of transport in multilayers with the current perpendicular to the interfaces was carried out and suggests that the right elemental combination is critical. In the case of our cluster-assembled materials, increasing the volume concentration of Co towards the percolation threshold while retaining the ultrafine magnetic structure or stabilizing the cluster magnetic moments by oxidation ${ }^{27}$ are also likely to increase the operating temperature.

In summary, this novel transport measurement carried out on magnetic cluster-assembled thin films shows a huge enhancement of its magnetic field response, about two orders of magnitude larger than the GMR ratio obtained in the same sample. This MTGV response is qualitatively different from GMR, both in its temperature, field and grain-size dependences. Hence, it is clear that a novel spin-dependent mechanism is involved, which cannot be reduced to some enhanced detection of the GMR effect and which is fundamentally different from that observed in multilayer systems. It is argued that the measurement method privileges the detection of the asymmetry of the spin-mixing process. This effect is shown to be extremely large in our cluster-assembled materials, when the magnetic structuring is refined to a nanometre scale.

\section{METHODS}

\section{SAMPLE PREPARATION}

A detailed description of the experimental setup used to generate the cluster-assembled samples and their characterization (X-ray photoelectron spectroscopy, high-resolution transmission electron microscopy, magnetic properties) has been published elsewhere ${ }^{15}$. Cobalt cluster ions of variable sizes are generated in a home-built magnetron cluster source following the design by Haberland et al. ${ }^{28}$. The mean size of the generated cluster distributions is adjustable between one atom and many thousands of atoms by varying the source conditions. Cluster ions are characterized by time-of-flight mass spectrometry and deposited on a substrate of slightly conductive polyimide foil (Kapton XC, Goodfellow, $40 \mu \mathrm{m}$ thickness) at room temperature. The deposition at energies well below $1 \mathrm{eV}$ per atom ensures soft-landing conditions, that is, fragmentation-free deposition ${ }^{29}$. The copper matrix is produced by electron beam evaporation and is deposited simultaneously with the clusters. In this study, samples were produced with mean cluster sizes of $\langle n\rangle=15 ; 600 ; 2,300$ and 6,500 atoms per cluster, the width of the size distributions $\sigma /\langle n\rangle$ varying between 0.6 and 0.16 for the smallest and largest clusters, respectively. Both the sample thickness $(50 \mathrm{~nm})$ and the concentration of $\sim 8$ at.\% Co were kept constant for all samples. A capping layer of $\sim 4 \mathrm{~nm} \mathrm{Cu}$ deposited on top of each sample ensures oxidation protection after exposure to air. The residual oxidation is discussed in ref. 15 .

We assume that our clusters remain intact on embedding because cobalt and copper are thermodynamically immiscible at room temperature. Furthermore, it has been shown that small Co islands on Cu surfaces retain their structural integrity and $\mathrm{Cu}$ capping is energetically favourable with respect to alloying ${ }^{30}$. 


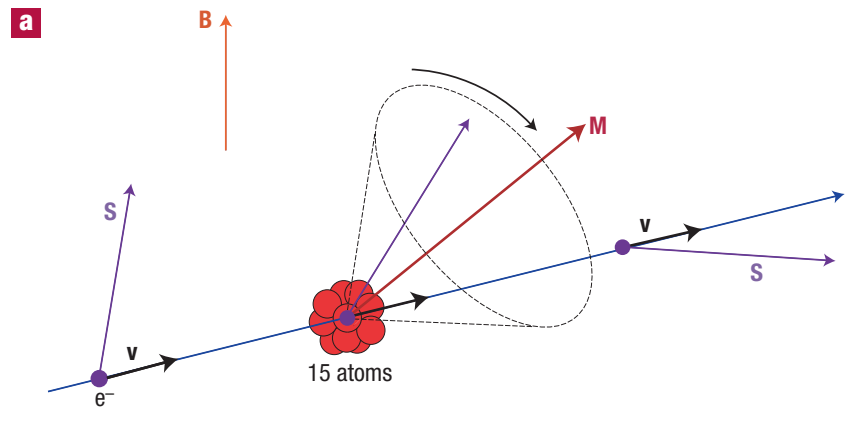

b
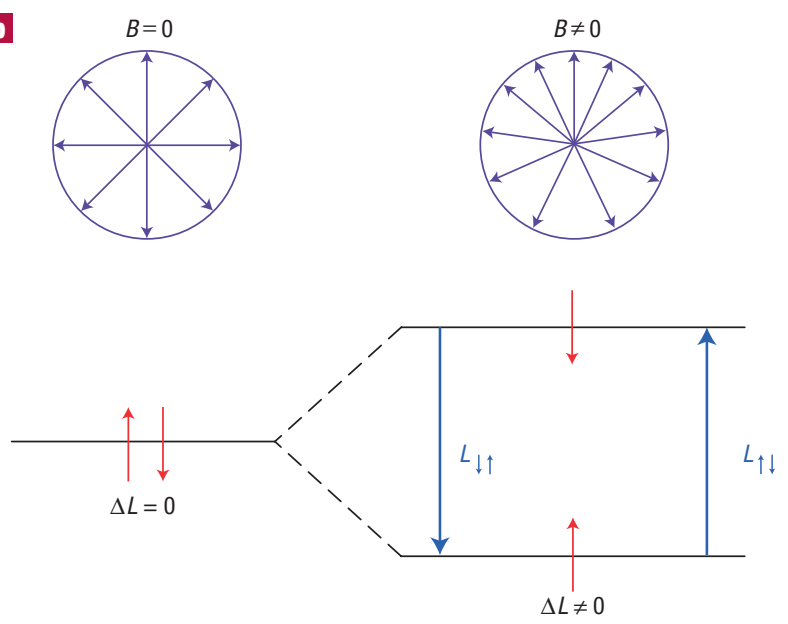

Figure 5 Jitterbug spin mixing. a, The precession of the conduction-electron spin about the exchange field in the magnetic cluster can change its orientation with respect to the external magnetic field. $\mathbf{b}$, A preferential orientation of the magnetic grains lifts the degeneracy of the two spin orientations, different transition rates between the two levels reflect the changed populations.

It has to be emphasized that our sample preparation is unique because it allows the independent control of both cluster size and concentration over a wide range.

\section{MTGV MEASUREMENTS}

The samples have an elongated shape: $20 \mathrm{~mm}$ long and $2 \mathrm{~mm}$ wide. The MTGV was measured with a direct current $I_{\text {d.c. }}$ of $8 \mathrm{~mA}$, corresponding to a current density of about $10^{4} \mathrm{~A} \mathrm{~cm}^{-2}$. Laser light, emitted by a $30 \mathrm{~mW}$ laser diode $(\lambda=680 \mathrm{~nm}$ ), and chopped at $22 \mathrm{~Hz}$, was used as a heat source (Fig. 2). The data points in Figs 3 and 4 are the average of the values obtained with the positive and negative field ramps. As the beam was not focused near the contacts, no net gradient of temperature was developed in the direction of the current. Consequently, the residual magnetothermoelectrical power response never exceeded $120 \mathrm{nV}$ and is thus negligible with respect to the MTGV.

We can exclude any spurious effect associated with the Hall effect because we observed about the same very large response for magnetic fields parallel (Fig. 2) and perpendicular to the film. We can also exclude a contribution of the Nernst effect ${ }^{31}$ because we find zero a.c. voltage drop when the current is zero (the Nernst effect is defined at zero current), and because the Nernst effect, like the Hall resistance, is an odd function of magnetic field.

Received 4 April 2006; accepted 17 July 2006; published 13 August 2006.

References

1. Baibich, M. N. et al. Giant magnetoresistance of (001) Fe/(001)Cr magnetic superlattices. Phys. Rev. Lett. 61, 2472-2475 (1988)

2. Binash, G., Grünberg, P., Saurenbach, F. \& Zinn, W. Enhanced magnetoresistance in layered magnetic structures with antiferromagnetic interlayer exchange. Phys. Rev. B 39, 4828-4830 (1989).

3. Berkowitz, A. E. et al. Giant magnetoresistance in heterogeneous Cu-Co alloys. Phys. Rev. Lett. 68, 3745-3748 (1992).

4. Parent, F. et al. Giant magnetoresistance in Co-Ag granular films prepared by low-energy cluster beam deposition. Phys. Rev. B 55, 3683-3687 (1997).

5. Binns, C. et al. The behaviour of nanostructured magnetic materials produced by depositing gas-phase nanoparticles. J. Phys. D: Appl. Phys. 38, R357-R379 (2005).

6. Parkin, S. S. P. et al. Giant tunnelling magnetoresistance at room temperature with $\mathrm{MgO}(100)$ tunnel barriers. Nature Mater. 3, 862-867 (2004).

7. Yuasa, S., Nagahama, T., Fukushima, A., Suzuki, Y. \& Ando, K. Giant room-temperature magnetoresistance in single-crystal $\mathrm{Fe} / \mathrm{MgO} / \mathrm{Fe}$ magnetic tunnel junctions. Nature Mater. 3, $868-871(2004)$

8. Moodera, J. S., Kinder, L. R., Wong, T. M. \& Meservey, R. Large magnetoresistance at room temperature in ferromagnetic thin film tunnel junctions. Phys. Rev. Lett. 74, 3273-3276 (1995).

9. Myers, E. B., Ralph, D. C., Katine, J. A., Louie, R. N. \& Buhrman, R. A. Current-induced switching of domains in magnetic multilayer devices. Science 285, 867-870 (1999).

10. Wegrowe, J.-E., Kelly, D., Jaccard, Y., Guittienne, Ph. \& Ansermet, J.-Ph. Current-induced magnetization reversal in magnetic nanowires. Europhys. Lett. 45, 626-632 (1999).

11. Urazhdin, S., Birge, N. O., Pratt, W. P. Jr \& Bass, J. Current-driven magnetic excitations in permalloy-based multilayer nanopillars. Phys. Rev. Lett. 91,146803 (2003).

12. Lee, K.-J., Deac, A., Redon, O., Nozières, J.-P. \& Dieny, B. Excitations of incoherent spin-waves due to spin-transfer torque. Nature Mater. 3, 877-881 (2004).

13. Kiselev, S. I. et al. Microwave oscillations of a nanomagnet driven by a spin-polarized current. Nature $425,380-383$ (2003).

14. Gravier, L., Serrano-Guisan, S., Reuse, F. \& Ansermet, J.-Ph. Thermodynamic description of heat and spin transport in magnetic nanostructures. Phys. Rev. B 73, 024419 (2006).

15. Hillenkamp, M., di Domenicantonio, G. \& Félix, C. Monodispersed metal clusters in solid matrices: A new experimental setup. Rev. Sci. Instrum. 77, 25104-25108 (2006).

16. Gregg, J. F., Allen, W., Thompson, S. M., Watson, M. L. \& Gehring, G. A. Jitterbug spin channel mixing in heterogeneous giant magnetoresistive material. J. Appl. Phys. 79, 5593-5595 (1996).

17. Gravier, L., Serrano-Guisan, S., Reuse, F. \& Ansermet, J.-Ph. Spin-dependent Peltier effect of perpendicular currents in multilayered nanowires. Phys. Rev. B 73, 052410 (2006).

18. Doudin, B., Blondel, A. \& Ansermet, J.-Ph. Arrays of multilayered nanowires. J. Appl. Phys. 79, 6090-6094 (1996).

19. Piraux, L. et al. Perpendicular magnetoresistance in Co/Cu multilayered nanowires. J. Magn. Magn. Mater. 156, 317-320 (1996).

20. Fert, A. \& Campbell, I. A. Two-current conduction in nickel. Phys. Rev. Lett. 21, 1190-1192 (1968).

21. Fert, A. Two-current conduction in ferromagnetic metals and spin wave-electron collisions. J. Phys. C (Solid State Phys.) 2, 1784-1788 (1969).

22. Piraux, L., Fert, A., Schroeder, P. A., Loloee, R. \& Etienne, P. Large magnetothermoelectric power in $\mathrm{Co} / \mathrm{Cu} \mathrm{Fe} / \mathrm{Cu}$ and $\mathrm{Fe} / \mathrm{Cr}$ multilayers. J. Magn. Magn. Mater. 110, L247-L253 (1992).

23. Piraux, L., Cassart, M., Jiang, J. S., Xiao, J. Q. \& Chien, C. L. Magnetothermal transport properties of granular Co-Ag solids. Phys. Rev. B 48, 638-641 (1993).

24. Gehring, G. A., Gregg, J. F., Thompson, S. M. \& Watson, M. L. Electron spin depolarisation in granular magnetic systems. J. Magn. Magn. Mater. 140-144, 501-502 (1995).

25. Cohen-Tannoudji et al. Quantum Mechanics (Wiley, Paris, complement $\mathrm{C}_{\mathrm{VV}}, 1977$ ).

26. Slichter, C. P. Principles of Magnetic Resonance (Springer Series in Solid-State Sciences, vol. 1, Springer, Berlin, 1996).

27. Skumryev, V. et al. Beating the superparamagnetic limit with exchange bias. Nature 423, 850-853 (2003).

28. Haberland, H. et al. Filling of micron-sized contact holes with copper by energetic cluster impact. J. Vac. Sci. Technol. A 12, 2925-2930 (1994).

29. Bromann, K. et al. Controlled deposition of size-selected silver nanoclusters. Science 274, 956-958 (1996).

30. Bernhard, T., Pfandzelter, R., Gruyters, M. \& Winter, H. P. Temperature-dependent intermixing of ultrathin Co films grown on $\mathrm{Cu}(100)$. Surf. Sci. 575, 154-162 (2005).

31. Wu, W., Lee, I. J. \& Chaikin, P. M. Giant Nernst effect and lock-in currents at magic angles in (TMTSF) ${ }_{2}$ PF $_{6}$. Phys. Rev. Lett. 91, 056601 (2003).

\section{Acknowledgements}

The authors gratefully acknowledge A. Fert for guiding us to the importance of spin mixing in our thermodynamical model, J. M. D. Coey for his interest in MTGV measurements and P. M. Chaikin for raising the question of the Nernst effect. The authors acknowledge the Swiss National Science Foundation for financial support.

Correspondence and requests for materials should be addressed to J.-P.A. or C.F.

Supplementary Information accompanies this paper on www.nature.com/naturematerials.

\section{Competing financial interests}

The authors declare that they have no competing financial interests.

Reprints and permission information is available online at http://npg.nature.com/reprintsandpermissions/ 